\title{
Sums of nonnegative multiplicative functions over integers without large prime factors II
}

by

Joung Min Song (Urbana, IL)

1. Introduction. In this paper we study the sums of multiplicative functions restricted to integers whose prime factors are small. Define the class $\mathcal{M}^{*}$ of nonnegative multiplicative functions by the following conditions: $h$ belongs to $\mathcal{M}^{*}$ if

- there exist constants $\delta, 0<\delta<1$, and $\kappa>1$ such that

$$
\sum_{p \leq z} h(p) \log p=\kappa z+O\left(z(\log z)^{-\delta}\right), \quad z \geq 2,
$$

- there exists a fixed constant $\varepsilon, 0<\varepsilon<1 / 2$, and a constant $b>0$ such that

$$
\sum_{p, k \geq 2} \frac{h\left(p^{k}\right)}{p^{k(1-\varepsilon)}} \leq b .
$$

Let $P(n)$ denote the largest prime divisor of a positive integer $n$, with $P(1)=1$. Our goal is to estimate the sum

$$
M(x, y):=\sum_{\substack{n \leq x \\ P(n) \leq y}} h(n)
$$

for $h \in \mathcal{M}^{*}$. We achieve this using a functional equation that is analogous to that of (1.4) and (3.6) of [S] (see (3.10) below) and using an inductive method originated in [Hi] and used also in [GM] and [S]. We express the estimate using a class of solutions to the differential difference equation (DDE) with delayed argument, namely,

$$
\left(u \varrho_{\kappa}(u)\right)^{\prime}=\kappa \varrho_{\kappa}(u)-\kappa \varrho_{\kappa}(u-1), \quad u>1,
$$

with initial conditions

$$
\varrho_{\kappa}(u)= \begin{cases}0, & u \leq 0, \\ \kappa B_{\kappa} u^{\kappa-1}, & 0<u \leq 1,\end{cases}
$$

2000 Mathematics Subject Classification: Primary 11N37. 
where $B_{\kappa}=e^{-\gamma \kappa} / \Gamma(\kappa+1)$, with $\gamma$ Euler's constant, and $\Gamma$ Euler's Gamma function. The function $\varrho_{\kappa}$ generalizes Dickman's function $\varrho$. Also, throughout the paper, we write

$$
u=\frac{\log x}{\log y} .
$$

MaIn Theorem. Suppose $h \in \mathcal{M}^{*}$. Then for all sufficiently large $y$ and for u satisfying

$$
1<u<(\log y)^{\delta / 2} / \log \log y
$$

we have

$$
M(x, y)=\frac{x}{\log y} V(y) \varrho_{\kappa}(u)\left\{1+O\left(\frac{\log (u+1)}{(\log y)^{\delta / 2}}\right)\right\}
$$

where

$$
V(y)=\prod_{p \leq y}\left(1+\sum_{k=1}^{\infty} \frac{h\left(p^{k}\right)}{p^{k}}\right) .
$$

The Main Theorem is related to the Main Theorem of $[\mathrm{dBvL}]$, II. By imposing a stronger set of conditions on the class of nonnegative multiplicative functions, an asymptotic estimate of $M(x, y)$ with error term is obtained here.

It is not hard to show that $\mathcal{M}^{*}$ is contained in a larger class $\mathcal{M}$ of multiplicative functions where for each $h \in \mathcal{M}$,

- there exist constants $\delta, 0<\delta<1$, and $\kappa>0$ such that

$$
\sum_{p \leq z} \frac{h(p)}{p} \log p=\kappa \log z+O\left((\log z)^{1-\delta}\right), \quad z \geq 2,
$$

- there exists a constant $b>0$ such that

$$
\sum_{p, k \geq 2} \frac{h\left(p^{k}\right)}{p^{k}} \log p^{k} \leq b .
$$

Previously, we established in $[\mathrm{S}]$ the estimate for the sum

$$
m(x, y):=\sum_{\substack{n \leq x \\ P(n) \leq y}} \frac{h(n)}{n} .
$$

Theorem 1. Suppose $h \in \mathcal{M}$. Then for all sufficiently large $y$,

$$
m(x, y)=V(y)\left\{j_{\kappa}(u)+O\left(\frac{\log (u+1)}{(\log y)^{\delta}}\right)\right\}
$$

uniformly for

$$
1 \leq u \leq \exp \left(\frac{1}{c}(\log y)^{\delta}\right)
$$


with $c$ a suitable positive constant, and $j_{\kappa}$ satisfying the DDE

$$
u j_{\kappa}^{\prime}(u)=\kappa j_{\kappa}(u)-\kappa j_{\kappa}(u-1), \quad u>1,
$$

and

$$
j_{\kappa}(u)= \begin{cases}0, & u \leq 0, \\ B_{\kappa} u^{\kappa}, & 0<u \leq 1,\end{cases}
$$

with $B_{\kappa}$ defined as in (1.2).

It was also shown in $[\mathrm{S}]$ that

$$
V(y)=\frac{C_{\kappa}}{B_{\kappa}}(\log y)^{\kappa}\left\{1+O\left(\frac{1}{(\log y)^{\delta}}\right)\right\},
$$

where

$$
C_{\kappa}=\frac{1}{\Gamma(\kappa+1)} \lim _{s \rightarrow 1+0} \prod_{p}\left(1+\sum_{k=1}^{\infty} \frac{h\left(p^{k}\right)}{p^{k s}}\right)\left(1-\frac{1}{p^{s}}\right)^{\kappa} .
$$

We note that

$$
\varrho_{k}(u)=j_{\kappa}^{\prime}(u), \quad u>0 .
$$

Theorem 1 was proved using the same inductive argument as in the proof of the Main Theorem, and was based on the following mean value theorem by $\mathrm{H}$. Halberstam for $h \in \mathcal{M}$.

TheOREm 2. For all sufficiently large $x$, and $h \in \mathcal{M}$,

$$
m(x):=\sum_{n \leq x} \frac{h(n)}{n}=C_{\kappa}(\log x)^{\kappa}+O\left((\log x)^{\kappa-\delta}\right) .
$$

In Section 3 we deduce the following consequence of Theorems 1 and 2.

Proposition 1. Suppose $h \in \mathcal{M}^{*}$. Then for all sufficiently large $y$,

$$
M(x, y)=\frac{x}{\log y} V(y)\left\{\varrho_{\kappa}(u)+O\left(\frac{\log (u+1)}{u(\log y)^{\delta}}\right)\right\}
$$

uniformly for the range of $u$ in Theorem 1.

It is easily seen that Proposition 1 is a much weaker version of the Main Theorem: It implies the validity of (1.4) for the range

$$
1<u \leq D \frac{\log \log y}{\log \log \log y},
$$

with a suitable constant $D$. The reason for the short $u$-range here is the faster-than-exponential-decrease in $\varrho_{\kappa}(u)$ (see Section 2). Nevertheless, (1.8) acts as the start of an iterative process that is the heart of the proof of the Main Theorem: Proposition 1 implies the Main Theorem for some initial range of $u$. In the proof of the Main Theorem, it is shown that the size of the error term cannot be reduced much further than stated. This is due also to the fast decrease of $\varrho_{\kappa}(u)$ as $u$ increases. 
2. About $\varrho_{\kappa}$. The solutions of differential difference equations of type (1.1) have been studied extensively by many authors (see for example [AO], $[\mathrm{GR}],[\mathrm{Hs}]$, and [Wh]). In this section, we look at several properties of $\varrho_{\kappa}(u)$ which will be crucial to proving the Main Theorem.

Lemma 1. For $\kappa>1$, we have the following properties of $\varrho_{\kappa}(u)$ :

(i) $\varrho_{\kappa}(u)=\exp \left\{-u \log u\left(1+O\left(\frac{\log \log u}{\log u}\right)\right)\right\}$ as $u \rightarrow \infty$.

(ii) $-\varrho_{\kappa}^{\prime}(u) / \varrho_{\kappa}(u)$ is an increasing function for $u>0$.

(iii) $-\varrho_{\kappa}^{\prime}(u) / \varrho_{\kappa}(u) \leq \log (2 u \log u)\left(u \geq e^{2}+1\right)$.

(iv) $\varrho_{\kappa}(u-t) / \varrho_{\kappa}(u) \leq(2 u \log u)^{t}$, uniformly for $u>e^{2}+1$ and $0<t \leq u$.

(v) For any positive number $\sigma$, we have

$$
x^{-\sigma} \ll \varrho_{\kappa}(u)
$$

for sufficiently large $y$ and $u_{0}<u<y^{\sigma / C}$, where $u_{0}$ depends on $C$, which derives from the $O$-constant in (i).

REMARKs. (1) In the proofs, we assume (i) and (ii): (i) is given (in different, but much stronger forms) in various places, for example in [Hs], and in $[\mathrm{Sm}]$, but for our present purpose this weak form suffices. In [Hs] Hensley gave an elementary proof of (ii) without using (i).

(2) Parts (iii) and (iv) are proved below with the same argument used in the proof of Lemma 1 in [Hi]. We remark that the lemma generalizes some of the properties of Dickman's $\varrho$ function. (The reader is referred to P. Moree's thesis $[\mathrm{M}]$ for much more information about the generalized Dickman function.)

(3) In (iv), the right side is replaced by a constant if $1<u<e^{2}+1$.

(4) We note that using a stronger form of (i) and Lemmas 4.3 and 4.4 of $[\mathrm{Sm}]$, one easily obtains

$$
\varrho_{\kappa}(u) \gg u^{-2 u}, \quad u>\kappa,
$$

which implies (v).

Proof. First, we prove (iii). We have, for all $\kappa>1$,

$$
u \varrho_{\kappa}(u)=\kappa \int_{0}^{1} \varrho_{k}(u-v) d v, \quad u>1,
$$

so that

$$
u=\kappa \int_{0}^{1} \frac{\varrho_{\kappa}(u-v)}{\varrho_{\kappa}(u)} d v=\kappa \int_{0}^{1} \exp \left(\int_{u-v}^{u} \frac{-\varrho_{\kappa}^{\prime}(s)}{\varrho_{\kappa}(s)} d s\right) d v
$$


For convenience, let $f(s)=-\varrho_{\kappa}^{\prime}(s) / \varrho_{\kappa}(s)$. Since $f(s)$ is nondecreasing for $s>0$ by (iii), we have

$$
u \geq \kappa \int_{0}^{1} \exp \{v f(u-1)\} d v=\kappa \frac{e^{f(u-1)}-1}{f(u-1)} .
$$

The rest of the proof relies on the fact that

$$
\phi_{\kappa}(x):=\kappa \frac{e^{x}-1}{x}
$$

is an increasing function of $x$ for all $x \in \mathbb{R}$. We have, on the one hand,

$$
u \geq \phi_{\kappa}(f(u-1)), \quad u>1,
$$

by (2.2). On the other hand, we have

$$
\begin{aligned}
\phi_{\kappa}(\log \{2(u-1) \log (u-1)\}) & =\kappa \frac{2(u-1) \log (u-1)-1}{\log (2(u-1) \log (u-1))} \\
& >\frac{2(u-1) \log (u-1)-1}{\log (2(u-1) \log (u-1))}
\end{aligned}
$$

for $\kappa>1$. If we show that the expression on the right of the last inequality is at least $u$, and thus, at least $\phi_{\kappa}(f(u-1))$, then we have the desired result by the monotonicity of $\phi_{\kappa}(\cdot)$. Indeed, setting $x=u-1$, we observe that

$$
2 x \log x-1 \geq(x+1) \log (2 x \log x)
$$

for $x \geq e^{2}$, since at $x=e^{2}$, we have

$$
4 e^{2}-1 \geq\left(e^{2}+1\right) \log \left(4 e^{2}\right),
$$

and the left hand side of (2.3) grows faster than the right hand side for $x \geq e^{2}$.

Now we use (iii) to prove (iv). First, suppose $u-t \geq e^{2}+1$. We have

$$
\begin{aligned}
\frac{\varrho_{\kappa}(u-t)}{\varrho_{\kappa}(u)} & =\exp \left\{\int_{u-t}^{u} f(s) d s\right\} \\
& \leq \exp \left\{\int_{u-t}^{u} \log (2 s \log s) d s\right\} \leq(2 u \log u)^{t},
\end{aligned}
$$

by (iii). If $u \geq e^{2}+1$ and $0 \leq u-t<e^{2}+1$, then

$$
\begin{aligned}
\frac{\varrho_{\kappa}(u-t)}{\varrho_{\kappa}(u)} & =\exp \left\{\int_{u-t}^{u} f(s) d s\right\} \\
& =\exp \left\{\int_{u-t}^{e^{2}+1} f(s) d s+\int_{e^{2}+1}^{u} f(s) d s\right\}
\end{aligned}
$$




$$
\begin{aligned}
& \leq \exp \left\{\int_{e^{2}+1}^{u} \log (2 s \log s) d s\right\} \exp \left\{\int_{u-t}^{e^{2}+1} f(s) d s\right\} \\
& \leq(2 u \log u)^{u-e^{2}-1} \exp \left\{\int_{u-t}^{e^{2}+1} f(s) d s\right\},
\end{aligned}
$$

by (iii). Since $f(\cdot)$ is increasing, the last expression is at most

$$
(2 u \log u)^{u-e^{2}-1} \cdot(2 u \log u)^{e^{2}+1-(u-t)}=(2 u \log u)^{t} .
$$

Part (v) is deduced using (i). Since

$$
\log \varrho_{\kappa}(u)=-u \log u\left\{1+O\left(\frac{\log \log u}{\log u}\right)\right\} \geq-C u \log u
$$

for a suitable constant $C$ and $u \geq u_{0}$, we have

$$
\varrho_{\kappa}\left(\frac{\log x}{\log y}\right) \geq x^{-C \frac{\log (\log x / \log y)}{\log y}} \geq x^{-\sigma}
$$

for any $\sigma>0$, provided

$$
\frac{\log x}{\log y}<y^{\sigma / C}
$$

Lemma 1(i) implies that $\varrho_{\kappa}(u) \rightarrow 0$ as $u \rightarrow \infty$. Also, from the definition we see that $\varrho_{\kappa}(u)$ increases on the range $0<u<1$ (for $\kappa>1$ ) and thus, there is at least one point $u>1$ at which $\varrho_{\kappa}$ achieves a maximum.

Proposition 2. Let $\kappa>1$. Then $\varrho_{\kappa}^{\prime}(u)$ has one and only one zero $u_{\kappa}$, and it lies between 1 and $\kappa$.

The proof was first given in [AO] using an elementary argument.

3. Proof of Proposition 1. In the current section we derive the functional equation under the conditions of $\mathcal{M}^{*}$. We prove several lemmas to express relationships between sums $m(x, y)$ and $M(x, y)$, and these will be used in the proof of Proposition 1.

3.1. Preliminary lemmas. By evaluating $\left(\Omega_{1}^{*}\right)$ at $p$ and $p-1$ respectively and taking the difference we deduce that

$$
h(p) \ll p(\log p)^{-1-\delta}
$$

for $h \in \mathcal{M}^{*}$. By Abel summation we get

$$
\begin{gathered}
\sum_{p \leq z} \frac{h(p)}{p}(\log p)^{1-\delta} \ll(\log z)^{1-\delta} \\
\sum_{p>z} \frac{h(p)}{p(\log p)^{\delta}} \ll(\log z)^{-\delta} .
\end{gathered}
$$


From (3.3) we see that

$$
\sum_{p} \frac{h(p)}{p(\log p)^{\delta}}<\infty .
$$

We observe that $\left(\Omega_{2}^{*}\right)$ implies not only $\left(\Omega_{2}\right)$, but also the following lemma.

LEMMA 2. If $h \in \mathcal{M}^{*}$ then

$$
\sum_{\substack{p^{k}>x \\ p, k \geq 2}} \frac{h\left(p^{k}\right)}{p^{k}} \log p^{k} \ll_{\varepsilon} x^{-\varepsilon / 2} .
$$

Proof. We prove that

$$
\sum_{\substack{p^{k}>x \\ p, k \geq 2}} \frac{h\left(p^{k}\right)}{p^{k}} \log p^{k} \leq b x^{-\varepsilon / 2}
$$

for $b$ and $\varepsilon$ as in $\left(\Omega_{2}^{*}\right)$, for all sufficiently large values of $x$, depending only on $\varepsilon$. Indeed, we have

$$
\begin{aligned}
& x^{\varepsilon / 2} \sum_{\substack{p^{k}>x \\
p, k \geq 2}} \frac{h\left(p^{k}\right)}{p^{k}} \log p^{k}<\sum_{\substack{p^{k}>x \\
p, k \geq 2}} \frac{h\left(p^{k}\right)}{p^{k}} p^{(\varepsilon / 2) k} \log p^{k} \\
& \leq \sum_{\substack{p^{k}>x \\
p, k \geq 2}} \frac{h\left(p^{k}\right)}{p^{k}} p^{k\left(\varepsilon / 2+\log \log p^{k} / \log p^{k}\right)} \\
& \leq \sum_{\substack{p, k \geq 2\\
}} \frac{h\left(p^{k}\right)}{p^{k(1-\varepsilon)}} \quad(\text { if } \log \log x / \log x \leq \varepsilon / 2) \\
& \leq b,
\end{aligned}
$$

by $\left(\Omega_{2}^{*}\right)$.

Lemma 3. Let $h \in \mathcal{M}^{*}$. Then

$$
M(x, y) \leq D x \frac{m(x, y)}{\log (e x)}, \quad x \geq 1,
$$

where $D$ is a suitable constant. In particular (taking $y \geq x$ ),

$$
M(x):=\sum_{n \leq x} h(n) \leq D x \frac{m(x)}{\log (e x)},
$$

where

$$
m(x):=\sum_{n \leq x} \frac{h(n)}{n} .
$$

REMARK. Inequality (3.7) is a little sharper than an exercise in [HaT], Chapter 0 . 
Proof. All we need here is the following weak consequence of $\left(\Omega_{1}^{*}\right)$ : There exists a constant $a$ such that

$$
\sum_{p \leq z} h(p) \log p \leq a z, \quad z \geq 1 .
$$

We have the equation

$$
M(x, y) \log x=\sum_{\substack{n \leq x \\ P(n) \leq y}} h(n) \log \frac{x}{n}+\sum_{\substack{n \leq x \\ P(n) \leq y}} h(n) \log n=T+S,
$$

say. Since $\log z \leq z-1$ for all $z>0$, we have

$$
0 \leq T \leq x m(x, y)-M(x, y) \text {. }
$$

Also,

$$
\begin{aligned}
S & =\sum_{\substack{m p^{k} \leq x \\
p \nmid m \\
P(m p) \leq y}} h(m) h\left(p^{k}\right) \log p^{k} \\
& \leq \sum_{\substack{m p \leq x \\
P(m p) \leq y}} h(m) h(p) \log p+\sum_{\substack{m p^{k} \leq x \\
k \geq 2 \\
P(m p) \leq y}} h(m) h\left(p^{k}\right) \log p^{k} \\
& \leq \sum_{\substack{m \leq x \\
P(m) \leq y}} h(m) \sum_{p \leq x / m} h(p) \log p+\sum_{\substack{p, k \geq 2 \\
p \leq y}} M\left(\frac{x}{p^{k}}, y\right) h\left(p^{k}\right) \log p^{k} \\
& \leq a x m(x, y)+\sum_{\substack{p, k \geq 2 \\
p \leq y}} \frac{x}{p^{k}} m\left(\frac{x}{p^{k}}, y\right) h\left(p^{k}\right) \log p^{k},
\end{aligned}
$$

by (3.9) and the obvious inequality $M(x, y) \leq x m(x, y)$ applied to the second sum. Hence, by $\left(\Omega_{2}\right)$,

$$
S \leq \operatorname{axm}(x, y)+b x m(x, y) .
$$

Therefore,

$$
M(x, y) \log (e x) \leq(1+a+b) x m(x, y)
$$

and this proves (3.6) with $D=1+a+b$. Inequality (3.7) restates (3.6) when $y \geq x$.

The above estimate of $M(x, y)$ is somewhat crude, nevertheless, it proves to be helpful for later use. More precisely, we can estimate $M(x, y)$ with an error term involving $m(x, y)$ as in the following lemma: 
Lemma 4. If $h \in \mathcal{M}^{*}$, then

$$
\begin{aligned}
M(x, y) \log x= & \sum_{\substack{m \leq x \\
P(m) \leq y}} h(m) \sum_{p \leq \min (x / m, y)} h(p) \log p \\
& +O\left(x m(x, y)(\log x)^{-\delta}\right), \quad x, y \geq 2 .
\end{aligned}
$$

In particular, when $y \geq x \geq 2$, we have

$$
M(x) \log x=\sum_{m \leq x} h(m) \sum_{p \leq x / m} h(p) \log p+O\left(x m(x)(\log x)^{-\delta}\right) .
$$

Proof. We start again from the identity (3.10). Suppose $2 \leq y \leq x$. We have

$$
S=\sum_{\substack{n \leq x \\ P(n) \leq y}} h(n) \log n=\sum_{\substack{m p^{k} \leq x \\ p \nmid m \\ P(m p) \leq y}} h(m) h\left(p^{k}\right) \log p^{k},
$$

and the main contribution to the sum $S$ comes from the terms corresponding to $k=1$, namely,

$$
\begin{gathered}
\sum_{\substack{m p \leq x \\
P(m p) \leq y \\
p \nmid m}} h(m) h(p) \log p=\sum_{\substack{m \leq x \\
P(m) \leq y}} h(m) \sum_{\substack{p \leq \min (x / m, y) \\
p \nmid m}} h(p) \log p \\
=\sum_{\substack{m \leq x \\
P(m) \leq y}} h(m) \sum_{p \leq \min (x / m, y)} h(p) \log p-\sum_{\substack{m p \leq x \\
p \mid m \\
P(m p) \leq y}} h(m) h(p) \log p .
\end{gathered}
$$

This gives

$$
\left|M(x, y) \log x-\sum_{\substack{m \leq x \\ P(m) \leq y}} h(m) \sum_{p \leq \min (x / m, y)} h(p) \log p\right|
$$$$
\leq T+\sum_{\substack{m p \leq x \\ p \mid m \\ P(m p) \leq y}} h(m) h(p) \log p+\sum_{\substack{m p^{k} \leq x \\ p, k \geq 2 \\ P(m p) \leq y}} h(m) h\left(p^{k}\right) \log p^{k}
$$

$$
=T+\sum_{\substack{l p^{k+1} \leq x \\ p \nmid l \\ P(l p) \leq y}} h(l) h\left(p^{k}\right) h(p) \log p+\sum_{\substack{m p^{k} \leq x \\ p, k \geq 2 \\ P(m p) \leq y}} h(m) h\left(p^{k}\right) \log p^{k}
$$

$$
\leq T+\sum_{\substack{k+1 \\ p^{k} \leq y}} M\left(\frac{x}{p^{k+1}}, y\right) h\left(p^{k}\right) h(p) \log p+\sum_{\substack{p, k \geq 2 \\ p \leq y}} M\left(\frac{x}{p^{k}}, y\right) h\left(p^{k}\right) \log p^{k} .
$$


Denote the two sums on the right side of (3.13) by $S^{\prime}$ and $S^{\prime \prime}$ respectively. We shall show that $S^{\prime}, S^{\prime \prime}$, and also $T$, have order of magnitude

$$
x m(x, y)(\log x)^{-\delta} .
$$

The terms in $S^{\prime}$ corresponding to $k=1$ contribute, by (3.6) and then (3.1), at most

$$
\begin{aligned}
x D \sum_{p \leq \sqrt{x}} \frac{m\left(x / p^{2}, y\right)}{\log \left(e x / p^{2}\right)} \cdot \frac{h(p)}{p(\log p)^{\delta}} & \\
& \ll \frac{x m(x, y)}{\log \left(e x^{1 / 3}\right)} \sum_{p \leq x^{1 / 3}} \frac{h(p)}{p(\log p)^{\delta}}+x m(x, y) \sum_{p>x^{1 / 3}} \frac{h(p)}{p(\log p)^{\delta}} \\
& \ll x m(x, y)(\log x)^{-\delta}
\end{aligned}
$$

by (3.4) and (3.3). The remaining terms of $S^{\prime}$ are, again by (3.6) and then (3.1), at most of order

$$
\begin{aligned}
& \frac{x m(x, y)}{\log (e \sqrt{x})} \sum_{\substack{p, k \geq 2 \\
p^{k+1} \leq \sqrt{x}}} \frac{h\left(p^{k}\right)}{p^{k+1}} h(p) \log p+x m(x, y) \sum_{\substack{p, k \geq 2 \\
\sqrt{x}<p^{k+1} \leq x}} \frac{h\left(p^{k}\right)}{p^{k+1}} \cdot \frac{h(p) \log p}{\log \left(e x / p^{k+1}\right)} \\
& \ll \frac{x m(x, y)}{\log (e \sqrt{x})} \sum_{\substack{p, k \geq 2 \\
p^{k+1} \leq \sqrt{x}}} \frac{h\left(p^{k}\right)}{p^{k}}(\log p)^{-\delta}+x m(x, y) \sum_{\substack{p, k \geq 2 \\
p^{k+1}>\sqrt{x}}} \frac{h\left(p^{k}\right)}{p^{k}}(\log p)^{-\delta} \\
& \ll \frac{x m(x, y)}{\log x}+x m(x, y) \sum_{\substack{p, k \geq 2 \\
p^{k}>x^{1 / 3}}} \frac{h\left(p^{k}\right)}{p^{k}}(\log p)^{-\delta} \\
& \ll \frac{x m(x, y)}{\log x}+\frac{x m(x, y)}{x^{\varepsilon / 6}} \ll \frac{x m(x, y)}{\log x}
\end{aligned}
$$

by $\left(\Omega_{2}^{*}\right)$ (and thus, by $\left(\Omega_{2}\right)$ ) and Lemma 2 . The second sum $S^{\prime \prime}$ is, for the same reasons, at most of order

$$
\begin{aligned}
& \frac{x}{\log (e \sqrt{x})} \sum_{\substack{p, k \geq 2 \\
p^{k} \leq \sqrt{x}}} m\left(\frac{x}{p^{k}}, y\right) \frac{h\left(p^{k}\right)}{p^{k}} \log p^{k}+x \sum_{\substack{p, k \geq 2 \\
p^{k}>\sqrt{x}}} m\left(\frac{x}{p^{k}}, y\right) \frac{h\left(p^{k}\right)}{p^{k}} \log p^{k} \\
& \ll \frac{x m(x, y)}{\log x}+\frac{x m(x, y)}{x^{\varepsilon / 4}} \ll \frac{x m(x, y)}{\log x} .
\end{aligned}
$$

Finally, we estimate $T$. We see that, by Lemma 3, 


$$
\begin{aligned}
T & =\sum_{\substack{n \leq x \\
P(n) \leq y}} h(n) \log \frac{x}{n}=\int_{1}^{x} M(t, y) \frac{d t}{t} \ll \int_{1}^{x} \frac{m(t, y)}{\log (e t)} d t \\
& \leq m(x, y) \int_{1}^{x} \frac{d t}{\log (e t)} \ll x \frac{m(x, y)}{\log x} .
\end{aligned}
$$

This proves the lemma.

3.2. Deduction of Proposition 1 from Theorem 1. The proof of Proposition 1 is obtained by combining Lemmas 3, 4 and Theorem 1. It is quite surprising that Proposition 1 is a rather straightforward consequence of Theorem 1.

From Lemma 4 we obtain

$$
\begin{aligned}
M(x, y) \log x= & \sum_{\substack{m \leq x / y \\
P(m) \leq y}} h(m) \sum_{p \leq y} h(p) \log p \\
& +\sum_{\substack{x / y<m \leq x / 2 \\
P(m) \leq y}} h(m) \sum_{p \leq x / m} h(p) \log p+O\left(x \frac{m(x, y)}{(\log x)^{\delta}}\right) .
\end{aligned}
$$

Note that in the second sum on the right the outer index $m$ runs only up to $x / 2$ since $p \geq 2$. We look at each of the three terms on the right side assuming, for the moment, $u \geq 2$. Consider the first sum on the right. By $\left(\Omega_{1}^{*}\right)$, it is equal to

$$
\sum_{\substack{m \leq x / y \\ P(m) \leq y}} h(m)\left\{\kappa y+O\left(y(\log y)^{-\delta}\right)\right\}=\kappa y M\left(\frac{x}{y}, y\right)\left\{1+O\left((\log y)^{-\delta}\right)\right\} .
$$

By Lemma 3, it is of order

$$
y M\left(\frac{x}{y}, y\right) \ll x \frac{m(x / y, y)}{\log y} \ll x \frac{V(y)}{\log y}, \quad u \geq 2,
$$

since

$$
m(x, y) \leq V(y)
$$

Applying $\left(\Omega_{1}^{*}\right)$ again to the second sum on the right of (3.14) we get

$$
\begin{aligned}
& \sum_{\substack{x / y<m \leq x / 2 \\
P(m) \leq y}} h(m)\left\{\kappa \frac{x}{m}+O\left(\frac{x}{m}\left(\log \frac{x}{m}\right)^{-\delta}\right)\right\} \\
= & \kappa x\{m(x / 2, y)-m(x / y, y)\}+O\left(\sum_{\substack{x / y<m \leq x / 2 \\
P(m) \leq y}} h(m) \frac{x}{m}\left(\log \frac{x}{m}\right)^{-\delta}\right) .
\end{aligned}
$$

The error term is at most of order 


$$
\sum_{\substack{x / y<m \leq x / 2 \\ P(m) \leq y}} h(m) \int_{3 / 2}^{x / m} \frac{d t}{(\log t)^{\delta}} \ll \int_{2}^{y} M\left(\frac{x}{t}, y\right) \frac{d t}{(\log t)^{\delta}},
$$

and by Lemma 3 it is, in turn, at most of order

$$
\begin{aligned}
x \int_{2}^{y} \frac{m(x / t, y)}{t \log (x / t)} \frac{d t}{(\log t)^{\delta}} & \ll x \frac{V(y)}{\log (x / y)} \int_{2}^{y} \frac{d t}{t(\log t)^{\delta}} \\
& \ll x \frac{V(y)}{\log y}(\log y)^{1-\delta} \ll x V(y)(\log y)^{-\delta}, \quad u \geq 2 .
\end{aligned}
$$

The main term on right side of (3.15) is equal to, by Theorem 1 ,

$$
\begin{aligned}
\kappa V(y) x\left(j_{\kappa}(u\right. & \left.\left.-\frac{\log 2}{\log y}\right)-j_{\kappa}(u-1)\right)+O\left(x V(y) \frac{\log (u+1)}{(\log y)^{\delta}}\right) \\
= & \kappa x V(y)\left(j_{\kappa}(u)-j_{\kappa}(u-1)\right) \\
& +O\left(x V(y)\left(j_{\kappa}(u)-j_{\kappa}\left(u-\frac{\log 2}{\log y}\right)+\frac{\log (u+1)}{(\log y)^{\delta}}\right)\right) \\
= & \kappa x V(y)\left(j_{\kappa}(u)-j_{\kappa}(u-1)\right)+O\left(x V(y) \frac{\log (u+1)}{(\log y)^{\delta}}\right)
\end{aligned}
$$

since

$$
j_{\kappa}(u)-j_{\kappa}\left(u-\frac{\log 2}{\log y}\right)=\int_{u-\log 2 / \log y}^{u} j_{\kappa}^{\prime}(t) d t \ll(\log y)^{-1} .
$$

Thus, we get

$$
M(x, y) \log x=\kappa x V(y)\left\{j_{\kappa}(u)-j_{\kappa}(u-1)\right\}+O\left(x V(y) \frac{\log (u+1)}{(\log y)^{\delta}}\right),
$$

which reduces, after division by $\log x$, to

$$
M(x, y)=x \frac{V(y)}{\log y}\left\{j_{\kappa}^{\prime}(u)+O\left(\frac{\log (u+1)}{u(\log y)^{\delta}}\right)\right\} .
$$

Now suppose $1 \leq u<2$. Again, (3.14) becomes

$$
\begin{aligned}
M(x, y) \log x= & \sum_{m \leq x / y} h(m) \sum_{p \leq y} h(p) \log p+\sum_{\substack{x / y \leq m \leq x / 2 \\
P(m) \leq y}} h(m) \sum_{p \leq x / m} h(p) \log p \\
& +O\left(x \frac{m(x, y)}{(\log x)^{\delta}}\right) \\
= & \sum_{m \leq x / y} h(m)\left\{\kappa y+O\left(y(\log y)^{-\delta}\right)\right\}
\end{aligned}
$$




$$
\begin{gathered}
+\sum_{\substack{x / y<m \leq x / 2 \\
P(m) \leq y}} h(m)\left\{\kappa \frac{x}{m}+\left(\frac{x}{m}\left(\log \frac{x}{m}\right)^{-\delta}\right)\right\}+O\left(x \frac{m(x, y)}{(\log x)^{\delta}}\right) \\
=\kappa x\left(m\left(\frac{x}{2}, y\right)-m\left(\frac{x}{y}, y\right)\right)+\kappa y M\left(\frac{x}{y}\right)\left(1+O\left((\log y)^{-\delta}\right)\right) \\
+O\left(x \frac{m(x, y)}{(\log x)^{\delta}}\right)+O\left(\sum_{\substack{x<m \leq x / 2 \\
P(m) \leq y}} h(m) \frac{x}{m}\left(\log \frac{x}{m}\right)^{-\delta}\right)
\end{gathered}
$$

by $\left(\Omega_{1}^{*}\right)$. We note that the last error term on the right side is at most of the same order as the error term on the right side of (3.15). Since $1<x / y \leq y$, Theorem 2 gives

$$
\begin{aligned}
m\left(\frac{x}{y}, y\right)=m\left(\frac{x}{y}\right) & =C_{\kappa} \log \left(\frac{x}{y}\right)^{\kappa}+O\left(\left(\log \left(\frac{x}{y}\right)\right)^{\kappa-\delta}\right) \\
& =V(y) B_{\kappa}(u-1)^{\kappa}+O\left((\log y)^{\kappa-\delta}\right) \\
& =V(y) j_{\kappa}(u-1)+O\left((\log y)^{\kappa-\delta}\right) \\
& =V(y)\left\{j_{\kappa}(u-1)+O\left(\frac{1}{(\log y)^{\delta}}\right)\right\}
\end{aligned}
$$

in the first term on the right, for $0 \leq u-1<1$. Together with Theorem 1 we can now deduce, as before, that

$$
\begin{aligned}
= & \kappa x V(y)\left\{j_{\kappa}(u)-j_{\kappa}(u-1)\right\}+O\left(x V(y)\left\{j_{\kappa}(u)-j_{\kappa}\left(u-\frac{\log 2}{\log y}\right)\right\}\right) \\
& +\kappa y M\left(\frac{x}{y}\right)\left(1+O\left((\log y)^{-\delta}\right)\right)+O\left(x V(y)\left\{\frac{\log (u+1)}{(\log y)^{\delta}}\right\}\right) \\
= & x V(y)\left\{u j_{\kappa}^{\prime}(u)+O\left(\frac{\log (u+1)}{(\log y)^{\delta}}+\frac{1}{\log y}\right)\right\} \\
& +\kappa y M\left(\frac{x}{y}\right)\left(1+O\left((\log y)^{-\delta}\right)\right) .
\end{aligned}
$$

To estimate the sum $M(\cdot)$ we use equations (3.12) and $\left(\Omega_{1}^{*}\right)$ :

$$
\begin{aligned}
M(w) \log w & =\sum_{m \leq w} h(m)\left\{\kappa \frac{w}{m}+O\left(\frac{w}{m}\left(\log \frac{e w}{m}\right)^{-\delta}\right)\right\}+O\left(w \frac{m(w)}{(\log w)^{\delta}}\right) \\
& =\kappa w \sum_{m \leq w} \frac{h(m)}{m}+O\left(w \frac{m(w)}{(\log w)^{\delta}}\right)+O\left(\int_{2}^{w} M\left(\frac{w}{t}\right) \frac{d t}{(\log e t)^{\delta}}\right) .
\end{aligned}
$$


The integral above is at most of order

$$
w \int_{2}^{w} \frac{m(w / t)}{t \log (e w / t)} \frac{d t}{(\log t)^{\delta}} \ll w(\log w)^{\kappa-\delta} \int_{0}^{1}(1-v)^{\kappa-1} v^{-\delta} d v
$$

by (3.7) and Theorem 2. Thus,

$$
M(w) \log w=w m(w)\left(\kappa+O\left((\log w)^{-\delta}\right)\right),
$$

and so

$$
M(w)=\kappa C_{\kappa} w(\log w)^{\kappa-1}+O\left(w(\log w)^{\kappa-1-\delta}\right) .
$$

This gives

$$
y M\left(\frac{x}{y}\right) \ll x \log \left(\frac{x}{y}\right)^{\kappa-1} \ll x V(y) \frac{(u-1)^{\kappa-1}}{\log y},
$$

and thus (3.17) reduces to

$$
M(x, y) \log x=x V(y)\left\{u j_{\kappa}^{\prime}(u)+O\left(\frac{(u-1)^{\kappa-1}}{\log y}+\frac{\log (u+1)}{(\log y)^{\delta}}\right)\right\} .
$$

After division by $\log x$ and recalling that $1 \leq u<2$, we get

$$
M(x, y)=x \frac{V(y)}{\log y}\left\{j_{\kappa}^{\prime}(u)+O\left(\frac{\log (u+1)}{u(\log y)^{\delta}}\right)\right\} .
$$

Since $j_{\kappa}^{\prime}(u)=\varrho_{\kappa}(u)$, this completes the proof of Proposition 1.

\section{Proof of the Main Theorem}

4.1. In this subsection, we establish the lemmas that will help us in proving the Main Theorem by an inductive method. For the remainder of the section, we assume

$$
\max \left(e^{2}+2, \kappa\right)<u \leq \frac{(\log y)^{\delta / 2}}{\log \log y}
$$

We have seen in Section 2 that in this range $\varrho_{\kappa}$ is monotonically decreasing.

Lemma 5. For $h \in \mathcal{M}^{*}$, and for a fixed $\theta, 1 / 2 \leq \theta \leq 1$, we have

$$
\begin{aligned}
& \sum_{p \leq y^{\theta}} \varrho_{\kappa}\left(u-\frac{\log p}{\log y}\right) \frac{h(p)}{p} \log p \\
& =\kappa \log y \cdot \int_{0}^{\theta} \varrho_{\kappa}(u-v) d v+O\left(\varrho_{\kappa}(u)(\log y)^{1-\delta / 2}\right),
\end{aligned}
$$

uniformly in u satisfying (4.1). 
Proof. We introduce the sum

$$
H(t):=\sum_{p \leq t} h(p) \log p=\kappa(t-2)+O\left(t(\log t)^{-\delta}\right), \quad t \geq 2,
$$

by $\left(\Omega_{1}^{*}\right)$. We can write the sum on the left of $(4.2)$ as

$$
\begin{aligned}
& \int_{2-}^{y^{\theta}} t^{-1} \varrho_{\kappa}\left(u-\frac{\log t}{\log y}\right) d H(t) \\
= & \int_{2-}^{y^{\theta}} t^{-1} \varrho_{\kappa}\left(u-\frac{\log t}{\log y}\right) d(H(t)-\kappa(t-2))+\kappa \int_{2}^{y^{\theta}} t^{-1} \varrho_{k}\left(u-\frac{\log t}{\log y}\right) d t .
\end{aligned}
$$

Using (4.3), we integrate the first integral on the right by parts to get

$$
\begin{aligned}
& \int_{2-}^{y^{\theta}} t^{-1} \varrho_{\kappa}\left(u-\frac{\log t}{\log y}\right) d H(t) \\
& =\kappa \int_{2}^{y^{\theta}} t^{-1} \varrho_{\kappa}\left(u-\frac{\log t}{\log y}\right) d t \\
& \quad+O\left(\frac{\varrho_{\kappa}(u-\theta)}{(\log y)^{\delta}}+\int_{2}^{y^{\theta}}\left\{\frac{\varrho_{\kappa}\left(u-\frac{\log t}{\log y}\right)}{t^{2}}+\left|\frac{\varrho_{\kappa}^{\prime}\left(u-\frac{\log t}{\log y}\right)}{t^{2} \log y}\right|\right\} \frac{t d t}{(\log t)^{\delta}}\right) .
\end{aligned}
$$

By Lemma 1(iii), the integral in the error term on the right of (4.5) is at most of order

$$
\int_{2}^{y^{\theta}} \frac{\varrho_{\kappa}\left(u-\frac{\log t}{\log y}\right)}{t(\log t)^{\delta}}\left\{1+\frac{\log (2 u \log u)}{\log y}\right\} d t \ll(\log y)^{1-\delta} \int_{\log 2 / \log y}^{\theta} \frac{\varrho_{\kappa}(u-v)}{v^{\delta}} d v
$$

uniformly on the interval (4.1). Using Lemma 1(iv), we have

$$
\begin{aligned}
(\log y)^{1-\delta} \int_{\log 2 / \log y}^{\theta} \frac{\varrho_{\kappa}(u-v)}{v^{\delta}} d v & \ll(\log y)^{1-\delta} \varrho_{\kappa}(u)(2 u \log u)^{\theta} \int_{0}^{\theta} \frac{d v}{v^{\delta}} \\
& \ll(\log y)^{1-\delta / 2} \varrho_{\kappa}(u)
\end{aligned}
$$

on the range (4.1). Also,

$$
\frac{\varrho_{\kappa}(u-\theta)}{(\log y)^{\delta}} \ll \varrho_{\kappa}(u) \frac{(2 u \log u)^{\theta}}{(\log y)^{\delta}} \ll \varrho_{\kappa}(u),
$$

if $u$ satisfies (4.1), by Lemma 1(iv). Therefore, from (4.5)-(4.7), we obtain

$$
\begin{aligned}
\int_{2-}^{y^{\theta}} t^{-1} \varrho_{\kappa}\left(u-\frac{\log t}{\log y}\right) d H(t)-\kappa \int_{2}^{y^{\theta}} t^{-1} \varrho_{\kappa}(u & \left.-\frac{\log t}{\log y}\right) d t \\
& \ll(\log y)^{1-\delta / 2} \varrho_{\kappa}(u) .
\end{aligned}
$$


Now we look at the second integral on the left of (4.8). Again using $v=$ $\log t / \log y$, we get

$$
\begin{aligned}
\kappa \int_{2}^{y^{\theta}} t^{-1} \varrho_{\kappa}\left(u-\frac{\log t}{\log y}\right) d t= & \kappa \log y \cdot \int_{\log 2 / \log y}^{\theta} \varrho_{\kappa}(u-v) d v \\
= & \kappa \log y \cdot \int_{0}^{\theta} \varrho_{\kappa}(u-v) d v \\
& +O\left(\log y \cdot \varrho_{\kappa}\left(u-\frac{\log 2}{\log y}\right) \int_{0}^{\log 2 / \log y} d v\right) \\
= & \kappa \log y \cdot \int_{0}^{\theta} \varrho_{\kappa}(u-v) d v+O\left(\varrho_{\kappa}(u)\right) .
\end{aligned}
$$

Therefore we arrive at

$\int_{2-}^{y^{\theta}} t^{-1} \varrho_{\kappa}\left(u-\frac{\log t}{\log y}\right) d H(t)=\kappa \log y \cdot \int_{0}^{\theta} \varrho_{\kappa}(u-v) d v+O\left(\varrho_{\kappa}(u)(\log y)^{1-\delta / 2}\right)$

on the range (4.1). This completes the proof of the lemma.

Lemma 6. Suppose $h \in \mathcal{M}^{*}$. Then for y sufficiently large we have

$$
\sum_{\substack{k+1 \\ p^{k} \leq y}} \varrho_{\kappa}\left(u-\frac{\log p^{k+1}}{\log y}\right) \frac{h\left(p^{k}\right)}{p^{k}} \cdot \frac{h(p)}{p} \log p \ll \varrho_{\kappa}(u)(u \log u),
$$

uniformly in u satisfying (4.1).

Proof. By Lemma 1(iv), we note that the sum is at most

$$
\varrho_{\kappa}(u) \sum_{\substack{p^{k+1} \leq x \\ p \leq y}} \frac{h\left(p^{k}\right)}{p^{k}} \cdot \frac{h(p)}{p} \log p \cdot(2 u \log u)^{\log p^{k+1} / \log y} .
$$

First, consider the sum of terms with $k=1$. It is at most of order

$$
\begin{aligned}
& \varrho_{\kappa}(u) \sum_{p \leq \min (\sqrt{x}, y)} \frac{h(p)}{p}(\log p)^{-\delta}(2 u \log u)^{\log p^{2} / \log y} \\
& \ll \varrho_{\kappa}(u) u \log u \sum_{p \leq \sqrt{y}} \frac{h(p)}{p}(\log p)^{-\delta}+\varrho_{\kappa}(u)(u \log u)^{2} \sum_{\sqrt{y}<p \leq y} \frac{h(p)}{p}(\log p)^{-\delta} \\
& \ll \varrho_{\kappa}(u)(u \log u)+\varrho_{\kappa}(u)(u \log u)^{2}(\log y)^{-\delta} \\
& \ll \varrho_{\kappa}(u)(u \log u),
\end{aligned}
$$


by (3.1), (3.3), and by (3.4) on the range (4.1). Now we look at the sum of terms in (4.10) with $k \geq 2$. They contribute at most

$$
\begin{aligned}
& \varrho_{\kappa}(u)(u \log u) \sum_{\substack{p^{k+1} \leq x \\
p \leq y}} \frac{h\left(p^{k}\right)}{p^{k}}(\log p)^{-\delta}(2 u \log u)^{k \log p / \log y} \\
& =\varrho_{\kappa}(u)(u \log u) \sum_{\substack{p^{k+1} \leq x \\
p \leq y}} h\left(p^{k}\right)(\log p)^{-\delta} p^{-k\{1-\log (2 u \log u) / \log y\}} .
\end{aligned}
$$

Since

$$
\begin{aligned}
\log (2 u \log u) & =\log 2+\log u+\log \log u \\
& \leq \log 2+\frac{\delta}{2} \log \log y+\log \log \log y
\end{aligned}
$$

we have

$$
\frac{\log (2 u \log u)}{\log y} \leq \delta \frac{\log \log y}{\log y}<\varepsilon
$$

in the range (4.1), and for all sufficiently large $y$. Thus, the sum in (4.11) is at most $\varrho_{\kappa}(u)(u \log u)$ by $\left(\Omega_{2}^{*}\right)$, and this completes the proof of the lemma.

Lemma 7. For $h \in \mathcal{M}^{*}$, we have

$$
\sum_{\substack{p, k \geq 2 \\ p^{k} \leq x \\ p \leq y}} \frac{h\left(p^{k}\right)}{p^{k}} \log p^{k} \cdot \varrho_{\kappa}\left(u-\frac{\log p^{k}}{\log y}\right) \ll \varrho_{\kappa}(u)(u \log u)
$$

for $u$ satisfying (4.1) and for all sufficiently large $y$.

Proof. We observe that

$$
\frac{1}{\varrho_{\kappa}(u)} \sum_{\substack{p, k \geq 2 \\ p^{k} \leq x \\ p \leq y}} \frac{h\left(p^{k}\right)}{p^{k}} \log p^{k} \cdot \varrho_{\kappa}\left(u-\frac{\log p^{k}}{\log y}\right) \ll \sum_{\substack{p, k \geq 2 \\ p^{k} \leq x \\ p \leq y}} \frac{h\left(p^{k}\right)}{p^{k}} \log p^{k} \cdot p^{k \alpha},
$$

where

$$
\alpha=\log (2 u \log u) / \log y
$$

by Lemma 1(iv). We separate the sum on the right side into two parts: one corresponding to $p^{k} \leq \log y$, and the other to $p^{k}>\log y$. The first part is at most of order

$$
\sum_{\substack{p, k \geq 2 \\ p^{k} \leq \log y}} \frac{h\left(p^{k}\right)}{p^{k}} \log p^{k} \cdot(\log y)^{\alpha} \leq b(2 u \log u)^{\log \log y / \log y} \ll u \log u
$$


by $\left(\Omega_{2}\right)$, for all sufficiently large values of $y$. For the part that corresponds to the condition $p^{k}>\log y$ we have

$$
\alpha+\log \log p^{k} / \log p^{k}<\alpha+\log \log y / \log y<\varepsilon
$$

for sufficiently large $y$, thus

$$
\sum_{\substack{p, k \geq 2 \\ p^{k}>\log y \\ p \leq y}} \frac{h\left(p^{k}\right)}{p^{k}} \log p^{k} \cdot p^{k \alpha} \ll \sum_{p, k \geq 2} \frac{h\left(p^{k}\right)}{p^{k(1-\varepsilon)}} .
$$

By $\left(\Omega_{2}^{*}\right)$ this is at most $O(1)$ and therefore,

$$
\sum_{\substack{p, k \geq 2 \\ p^{k} \leq x \\ p \leq y}} \frac{h\left(p^{k}\right)}{p^{k}} \log p^{k} \cdot p^{k \alpha} \ll u \log u,
$$

giving us the claim.

LEMma 8. We have

$$
\int_{1}^{x} \varrho_{\kappa}\left(\frac{\log t}{\log y}\right) d t \ll x \varrho_{\kappa}(u),
$$

uniformly for

$$
1<u \leq \sqrt{y}
$$

Proof. Once again, if we let $v=\log t / \log y$, the integral in the lemma becomes

$$
\begin{aligned}
\log y \cdot \int_{0}^{u} \varrho_{\kappa}(v) y^{v} d v & =x \log y \cdot \int_{0}^{u} \varrho_{\kappa}(u-v) y^{-v} d v \\
& =\varrho_{\kappa}(u) x \log y \cdot \int_{0}^{u} \frac{\varrho_{\kappa}(u-v)}{\varrho_{\kappa}(u)} y^{-v} d v \\
& \ll \varrho_{\kappa}(u) x \log y \cdot \int_{0}^{u}\left(\frac{2 u \log u}{y}\right)^{v} d v
\end{aligned}
$$

by Lemma 1(iv). If $u$ satisfies (4.13), this is $\ll x \varrho_{\kappa}(u)$.

4.2. Proof of the Main Theorem. The proof of the Main Theorem is analogous to that of Theorem 1 (see $[\mathrm{S}]$ ), but is more delicate because of the rapid decline of $\varrho_{\kappa}(u)$ as $u \rightarrow \infty$.

For $u \geq 1$, define $\Delta(u, y)$ by the following formula:

$$
M(x, y)=\frac{x}{\log y} V(y) \varrho_{\kappa}(u)\{1+\Delta(u, y)\} .
$$

We have, a priori,

$$
\Delta(u, y)=o(1)
$$


as $y \rightarrow \infty$ for the small range (1.9), by virtue of Proposition 1 ; in particular, for any positive fixed $u_{0}$, there exists $K=K\left(u_{0}\right)$ such that we have

$$
|\Delta(u, y)| \leq K \frac{\log (u+1)}{(\log y)^{\delta / 2}}, \quad 1<u \leq u_{0},
$$

from the fact that the function $\varrho_{\kappa}$ is bounded from below for small values of $u$. This proves the Main Theorem in the range $1 \leq u \leq u_{0}$. Let

$$
\Delta^{*}(u, y):=\sup _{1<u^{\prime} \leq u}\left|\Delta\left(u^{\prime}, y\right)\right| .
$$

Then our goal here is to prove

$$
\Delta^{*}(u, y) \ll \frac{\log (u+1)}{(\log y)^{\delta / 2}}
$$

for all sufficiently large values of $y$ and for a range of $u$ greater than (1.9). Here, we again use (3.13), and estimate each of $T, S^{\prime}$ and $S^{\prime \prime}$ as well as the term on the left side of (3.13) for all

$$
u \geq u_{0}=\max \left(e^{2}+2, \kappa+2\right),
$$

with $\Delta(u, y)$ defined by (4.14). Suppose we are in the range (4.1). First, we write

$$
T=\sum_{\substack{n \leq x \\ P(n) \leq y}} h(n) \log \frac{x}{n}=\int_{1}^{x} M(t, y) \frac{d t}{t}=\int_{1}^{y} M(t) \frac{d t}{t}+\int_{y}^{x} M(t, y) \frac{d t}{t} .
$$

We deduce that the first integral on the right is at most of order

$$
\begin{aligned}
\int_{1}^{y}(\log t)^{\kappa-1} d t & \ll y(\log y)^{\kappa} \ll y V(y)=\frac{x}{\log y}\left(\frac{x}{y \log y}\right)^{-1} V(y) \\
& \ll \frac{x V(y)}{\log y} x^{-1 / 2} \ll \frac{x V(y)}{\log y} \varrho_{\kappa}(u),
\end{aligned}
$$

by (3.18), (1.6), and Lemma $1(\mathrm{v})$, for the range (4.1). The second integral is equal to, with (4.14),

$$
\begin{aligned}
\int_{y}^{x} \frac{t}{\log y} V(y) \varrho_{\kappa}\left(\frac{\log t}{\log y}\right)\left(1+\Delta\left(\frac{\log t}{\log y}, y\right)\right) \frac{d t}{t} & \\
\leq & \frac{V(y)}{\log y} \int_{y}^{x} \varrho_{\kappa}\left(\frac{\log t}{\log y}\right) d t\left(1+\Delta^{*}(u, y)\right) \ll \frac{x V(y)}{\log y} \varrho_{\kappa}(u)\left(1+\Delta^{*}(u, y)\right)
\end{aligned}
$$

by Lemma 8 . Thus,

$$
T \ll \frac{x V(y)}{\log y} \varrho_{\kappa}(u)\left(1+\Delta^{*}(u, y)\right) .
$$


Now turn to $S^{\prime \prime}$. We have

$$
\begin{aligned}
S^{\prime \prime} & =\sum_{\substack{p^{k} \leq x \\
p \leq y \\
k \geq 2}} M\left(\frac{x}{p^{k}}, y\right) h\left(p^{k}\right) \log p^{k} \\
& =\sum_{\substack{p^{k} \leq x / y \\
p \leq y \\
k \geq 2}} M\left(\frac{x}{p^{k}}, y\right) h\left(p^{k}\right) \log p^{k}+\sum_{\substack{x / y<p^{k} \leq x \\
p \leq y \\
k \geq 2}} M\left(\frac{x}{p^{k}}\right) h\left(p^{k}\right) \log p^{k} .
\end{aligned}
$$

The first sum is at most of order

$$
\begin{aligned}
& \frac{x V(y)}{\log y} \sum_{\substack{p^{k} \leq x / y \\
p \leq y, k \geq 2}} \frac{h\left(p^{k}\right)}{p^{k}} \log p^{k} \cdot \varrho_{\kappa}\left(u-\frac{\log p^{k}}{\log y}\right)\left(1+\Delta^{*}(u, y)\right) \\
& \ll \frac{x V(y)}{\log y} \varrho_{\kappa}(u)(2 u \log u)\left(1+\Delta^{*}(u, y)\right)
\end{aligned}
$$

by Lemma 7 . The second sum in $S^{\prime \prime}$ is at most

$$
x m(y) \sum_{\substack{x / y<p^{k} \leq x \\ p \leq y \\ k \geq 2}} \frac{h\left(p^{k}\right)}{p^{k}} \log p^{k} \ll x V(y)\left(\frac{x}{y}\right)^{-\varepsilon / 2},
$$

first by Lemma 3 , then by Lemma 2 . Since $x / y>\sqrt{x}$,

$$
\left(\frac{x}{y}\right)^{-\varepsilon / 2} \ll x^{-\varepsilon / 4} \ll \frac{x^{-\varepsilon / 5}}{\log y} \ll \frac{\varrho_{\kappa}(u)}{\log y},
$$

for all $y$ sufficiently large due to Lemma $1(\mathrm{v})$. Similarly, we see that

$$
\begin{aligned}
& S^{\prime}=\sum_{\substack{p^{k+1} \leq x / y \\
p \leq y}} M\left(\frac{x}{p^{k+1}}, y\right) h\left(p^{k}\right) h(p) \log p \\
& +\sum_{\substack{x / y<p^{k+1} \leq x \\
p \leq y}} M\left(\frac{x}{p^{k+1}}\right) h\left(p^{k}\right) h(p) \log p \\
& \ll \frac{x V(y)}{\log y} \sum_{p^{k+1} \leq x} \frac{h\left(p^{k}\right)}{p^{k}} \cdot \frac{h(p)}{p} \log p \cdot \varrho_{\kappa}\left(u-\frac{\log p^{\kappa+1}}{\log y}\right)\left(1+\Delta^{*}(u, y)\right) \\
& +x V(y) \sum_{\substack{p^{k+1}>x / y \\
p \leq y}} \frac{h\left(p^{k}\right)}{p^{k}} \cdot \frac{h(p)}{p} \log p .
\end{aligned}
$$


By Lemma 6, we see that the first sum on the right side of (4.16) is of the order

$$
x \frac{V(y)}{\log y} \varrho_{\kappa}(u)(u \log u)\left(1+\Delta^{*}(u, y)\right) \ll x \frac{V(y)}{\log y} \varrho_{\kappa}(u)(\log y)^{\delta / 2}\left(1+\Delta^{*}(u, y)\right) .
$$

Also, the second sum is, by (3.1), at most

$$
\begin{aligned}
x V(y) \sum_{\substack{p^{k+1}>x / y \\
p \leq y}} \frac{h\left(p^{k}\right)}{p^{k}}(\log p)^{-\delta} & \left.\leq x V(y) \sum_{\substack{p^{k} \geq x / y^{2} \\
p \leq y, k \geq 2}} \frac{h\left(p^{k}\right)}{p^{k}} \quad \text { (if } u \geq 3\right) \\
& \ll x V(y)\left(\frac{x}{y^{2}}\right)^{-\varepsilon / 2} \quad \text { (by Lemm } \\
& \ll x V(y)(\log y)^{-\delta / 2} \varrho_{\kappa}(u),
\end{aligned}
$$

again by Lemma $1(\mathrm{v})$. Therefore, from (3.13) we have

$$
\begin{aligned}
M(x, y) \log x= & \sum_{\substack{m \leq x \\
P(m) \leq y}} h(m) \sum_{p \leq \min (x / m, y)} h(p) \log p \\
& +O\left(\frac{x V(y)}{\log y} \varrho_{\kappa}(u)(\log y)^{1-\delta / 2}\left(1+\Delta^{*}(u, y)\right)\right) .
\end{aligned}
$$

Denote the sum on the right side of (4.17) by $S_{M}$. By changing the order of summation, we come to

$$
\begin{aligned}
S_{M} & =\sum_{p \leq y}\left(\sum_{\substack{m \leq x / p \\
P(m) \leq y}} h(m)\right) h(p) \log p=\sum_{p \leq y} M\left(\frac{x}{p}, y\right) h(p) \log p \\
& =x \frac{V(y)}{\log y} \sum_{p \leq y} \frac{h(p)}{p} \log p \cdot \varrho_{\kappa}\left(u-\frac{\log p}{\log y}\right)\left\{1+\Delta\left(u-\frac{\log p}{\log y}, y\right)\right\} .
\end{aligned}
$$

The main term in the summation equals, by Lemma 5 with $\theta=1$,

$$
\kappa \log y \cdot \int_{0}^{1} \varrho_{\kappa}(u-v) d v+O\left(\varrho_{\kappa}(u)(\log y)^{1-\delta / 2}\right) .
$$

Thus,

$$
\begin{aligned}
S_{M}= & \kappa x V(y) \int_{0}^{1} \varrho_{\kappa}(u-v) d v \\
& +x \frac{V(y)}{\log y}\left\{\sum_{p \leq y^{1 / 2}} \frac{h(p)}{p} \log p \cdot \varrho_{\kappa}\left(u-\frac{\log p}{\log y}\right) \Delta\left(u-\frac{\log p}{\log y}, y\right)\right.
\end{aligned}
$$




$$
\begin{aligned}
& +\sum_{y^{1 / 2}<p \leq y} \frac{h(p)}{p} \log p \cdot \varrho_{\kappa}\left(u-\frac{\log p}{\log y}\right) \Delta\left(u-\frac{\log p}{\log y}, y\right) \\
& \left.+O\left(\varrho_{\kappa}(u)(\log y)^{1-\delta / 2}\right)\right\} .
\end{aligned}
$$

Applying Lemma 5 with $\theta=1 / 2$ and $\theta=1$ respectively to the two sums on the right side, we get from (4.17),

$$
\begin{aligned}
\left|M(x, y) \log x-\kappa x V(y) \int_{0}^{1} \varrho_{\kappa}(u-v) d v\right| \\
\leq x \frac{V(y)}{\log y}\left\{\kappa \log y \cdot \int_{0}^{1 / 2} \varrho_{\kappa}(u-v) d v \Delta^{*}(u, y)\right. \\
+\kappa \log y \cdot \int_{1 / 2}^{1} \varrho_{\kappa}(u-v) d v \Delta^{*}(u-1 / 2, y) \\
\left.+O\left(\varrho_{\kappa}(u)\left(1+\Delta^{*}(u, y)\right)(\log y)^{1-\delta / 2}\right)\right\} .
\end{aligned}
$$

Dividing both sides of the equation (4.18) by $x V(y) \varrho_{\kappa}(u) u$, and using (2.1) we infer that

$$
\begin{aligned}
& |\Delta(u, y)| \\
& \leq \frac{\kappa}{u \varrho_{\kappa}(u)}\left\{\int_{0}^{1 / 2} \varrho_{\kappa}(u-v) d v \Delta^{*}(u, y)+\int_{1 / 2}^{1} \varrho_{\kappa}(u-v) d v \Delta^{*}(u-1 / 2, y)\right\} \\
& +O\left(\frac{1+\Delta^{*}(u, y)}{u(\log y)^{\delta / 2}}\right),
\end{aligned}
$$

and we write the right side as

$$
I(u) \Delta^{*}(u, y)+(1-I(u)) \Delta^{*}(u-1 / 2, y)+O\left(\frac{1+\Delta^{*}(u, y)}{u(\log y)^{\delta / 2}}\right),
$$

where

$$
I(u):=\frac{\kappa}{u \varrho_{\kappa}(u)} \int_{0}^{1 / 2} \varrho_{\kappa}(u-v) d v
$$

We note that since $\varrho_{\kappa}$ is decreasing on the range $(4.1)$, we have

$$
\int_{0}^{1 / 2} \varrho_{\kappa}(u-v) d v<\int_{1 / 2}^{1} \varrho_{\kappa}(u-v) d v
$$


and hence

$$
I(u) \leq \frac{\kappa}{2 u \varrho_{\kappa}(u)}\left(\int_{0}^{1 / 2} \varrho_{\kappa}(u-v) d v+\int_{1 / 2}^{1} \varrho_{\kappa}(u-v) d v\right)=1 / 2 .
$$

Using (4.19), we observe that

$$
\begin{gathered}
\frac{1}{2}\left(\Delta^{*}(u, y)+\Delta^{*}(y, u-1 / 2)\right)-\left(I(u) \Delta^{*}(u, y)+(1-I(u)) \Delta^{*}(u-1 / 2, y)\right) \\
=(1 / 2-I(u))\left(\Delta^{*}(u, y)-\Delta^{*}(u-1 / 2, y)\right) \geq 0,
\end{gathered}
$$

by the monotonicity of $\Delta^{*}(u, y)$, for all

$$
u_{0} \leq u \leq(\log y)^{\delta / 2} / \log \log y .
$$

From this we deduce the inequality

$$
\begin{array}{r}
|\Delta(u, y)| \leq \frac{1}{2}\left(\Delta^{*}(u, y)+\Delta^{*}(u-1 / 2, y)\right)+O\left(\frac{1+\Delta^{*}(u, y)}{u(\log y)^{\delta / 2}}\right) \\
u_{0} \leq u \leq(\log y)^{\delta / 2} / \log \log y .
\end{array}
$$

Next, we show that (4.20) still holds if we replace $\Delta(u, y)$ on the left side by $\Delta^{*}(u, y)$ : Suppose $u-1 / 2 \leq u^{\prime} \leq u$. Then (4.20) gives

$$
\begin{aligned}
\left|\Delta\left(u^{\prime}, y\right)\right| & \leq \frac{1}{2}\left(\Delta^{*}\left(u^{\prime}, y\right)+\Delta^{*}\left(u^{\prime}-1 / 2, y\right)\right)+O\left(\frac{1+\Delta^{*}\left(u^{\prime}, y\right)}{u^{\prime}(\log y)^{\delta / 2}}\right) \\
& \leq \frac{1}{2}\left(\Delta^{*}(u, y)+\Delta^{*}(u-1 / 2, y)\right)+O\left(\frac{1+\Delta^{*}(u, y)}{u(\log y)^{\delta / 2}}\right),
\end{aligned}
$$

by the monotonicity of $\Delta^{*}(u, y)$, where the $O$ constant changes at most by a factor of $3 / 2$. On the other hand, if $u_{0} \leq u^{\prime}<u-1 / 2$, then

$$
\left|\Delta\left(u^{\prime}, y\right)\right| \leq \Delta^{*}(u-1 / 2, y) \leq \frac{1}{2}\left(\Delta^{*}(u, y)+\Delta^{*}(u-1 / 2, y)\right) .
$$

Thus, it follows that uniformly for $u_{0} \leq u \leq(\log y)^{\delta / 2} /(2 \log \log y)$, we have

$$
\Delta^{*}(u, y) \leq \frac{1}{2}\left(\Delta^{*}(u, y)+\Delta^{*}(u-1 / 2, y)\right)+O\left(\frac{1+\Delta^{*}(u, y)}{u(\log y)^{\delta / 2}}\right) .
$$

We rearrange terms to arrive at

$$
\Delta^{*}(u, y) \leq \Delta^{*}(u-1 / 2, y)+O\left(\frac{1+\Delta^{*}(u, y)}{u(\log y)^{\delta / 2}}\right),
$$

and iterate with respect to $u$ to get

$$
\Delta^{*}(u, y) \leq \Delta^{*}\left(v_{0}, y\right)+O\left(\frac{\left(1+\Delta^{*}(u, y)\right) \log u}{(\log y)^{\delta / 2}}\right),
$$

where

$$
u_{0}-1 / 2 \leq v_{0} \leq u_{0} .
$$


By (4.15), we have

$$
\Delta^{*}(u, y) \ll \frac{\left(1+\Delta^{*}(u, y)\right) \log u}{(\log y)^{\delta / 2}}, \quad u \geq u_{0} .
$$

Thus, there exists a constant $B$ such that

$$
\Delta^{*}(u, y) \leq B \frac{\left(1+\Delta^{*}(u, y)\right) \log u}{(\log y)^{\delta / 2}}
$$

and

$$
\Delta^{*}(u, y)\left(1-\frac{B \log u}{(\log y)^{\delta / 2}}\right) \leq \frac{B \log u}{(\log y)^{\delta / 2}}
$$

This yields

$$
\Delta^{*}(u, y) \ll \frac{\log u}{(\log y)^{\delta / 2}}\left(1+B^{\prime} \frac{\log u}{(\log y)^{\delta / 2}}\right),
$$

for an appropriate $B^{\prime}$, and therefore

$$
\Delta^{*}(u, y) \ll \frac{\log u}{(\log y)^{\delta / 2}}
$$

in the range (4.1). This completes the proof of the theorem.

Acknowledgements. The author wishes to express her gratitude to Professor H. Halberstam for his many ideas and comments.

\section{References}

[AO] N. C. Ankeny and H. Onishi, The general sieve, Acta Arith. 10 (1964), 31-62.

[dBvL] N. G. de Bruijn and J. H. van Lint, Incomplete sums of multiplicative functions, I, II, Indag. Math. 26 (1964), 339-347; 348-359.

[GM] D. A. Goldston and K. S. McCurley, Sieving the positive integers by large primes, J. Number Theory 28 (1988), 94-115.

[GR] F. Grupp and H.-E. Richert, Notes on functions connected with the sieve, Analysis 8 (1988), 1-23.

[HaT] R. Hall and G. Tenenbaum, Divisors, Cambridge Univ. Press, 1988.

[Hs] D. Hensley, The convolution power of the Dickman function, J. London Math. Soc. (2) 33 (1986), 395-406.

[Hi] A. Hildebrand, On the number of positive integers $\leq x$ and free of prime factors $>$ y, J. Number Theory 22 (1986), 289-307.

[M] P. Moree, Psixyology and Diophantine equations, thesis, Leiden University, 1993.

[Sm] H. Smida, Valeur moyenne des fonctions de Piltz sur les entiers sans grand facteur premier, Acta Arith. 63 (1993), 21-50.

[S] J. M. Song, Sums of nonnegative multiplicative functions over integers without large prime factors I, ibid. 97 (2001), 329-351. 
[Wh] F. Wheeler, On two differential difference equations arising in analytic number theory, PhD thesis, University of Illinois, 1988.

Department of Mathematics

University of Illinois

1409 W. Green Street

Urbana, IL 61801, U.S.A.

E-mail: song@math.uiuc.edu

Received on 8.12.1999

and in revised form on 12.6.2001 